\title{
Real-time identification of gastric lesions and anatomical landmarks by artificial intelligence during magnetically controlled capsule endoscopy
}

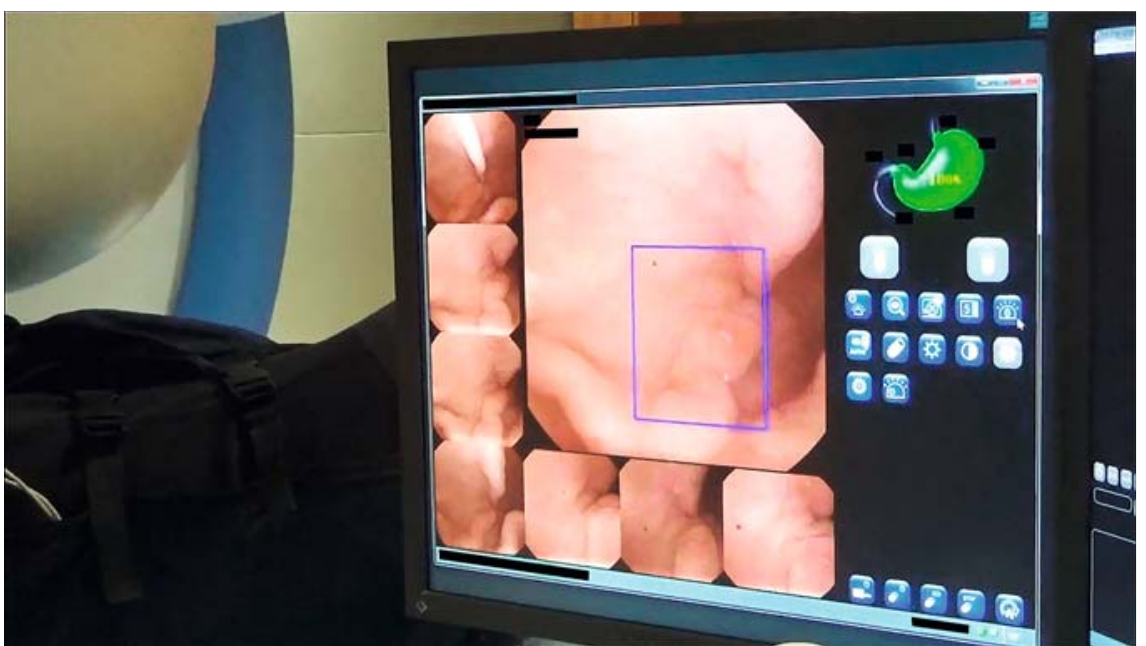

- Fig. 1 Monitor interface of the artificial intelligence-based real-time diagnostic system in magnetically controlled capsule endoscopy.

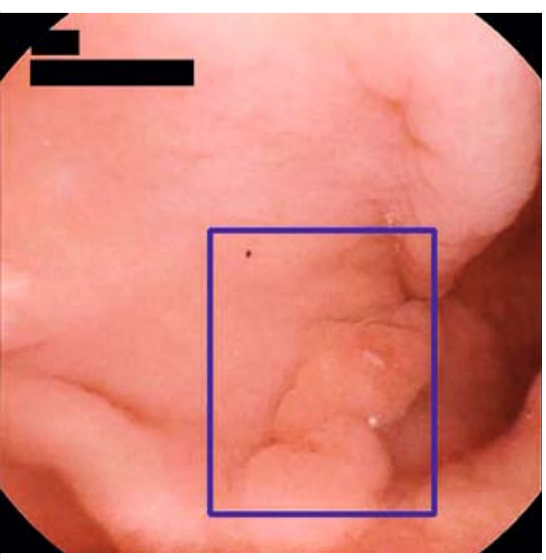

Fig. 2 Real-time identification of erosion/bleeding/ulcer (blue frame) in the gastric antrum.

Artificial intelligence $(\mathrm{Al})$ has revolutionized the diagnosis of gastrointestinal endoscopy, including capsule endoscopy. In our previous study, we developed and validated an Al-based auxiliary system for diagnosing gastric lesions based on still images [1]. Here, we demonstrate the performance of the first Al-based real-time diagnostic system in magnetically controlled capsule endoscopy (MCE) for detecting gastric lesions

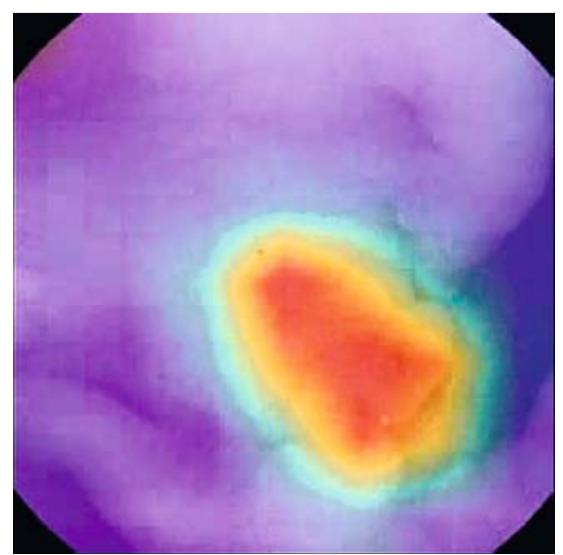

- Fig. 3 Lesion detection by heat map in the gastric antrum.

(Smart Data Service System-AI [SDSSAl]; Ankon Technologies Co., Ltd., Wuhan, China) (॰ Fig. 1).

A total of 34062 MCE images from 856 patients treated at Changhai Hospital from January 2016 to October 2019 were used to train the SDSS-AI system. In addition, 50 patients referred for MCE at Changhai Hospital from December 2019 to January 2020 were enrolled to evaluate the diagnostic accuracy of SDSS-AI, using expert readings as the gold standard. Overall sensitivity of SDSS-AI for detecting gastric lesions was $98.9 \%$ (95\% confidence interval $[\mathrm{Cl}], 93.3 \%-99.9 \%)$, with sensitivities of $98.7 \%(95 \% \mathrm{Cl} 91.9 \%-$ $99.9 \%)$ and $100 \%(95 \% \mathrm{Cl} 77.1 \%-100 \%)$ for detecting gastric erosion/bleeding/ ulcer and polyp/submucosal tumor, respectively (>Fig.2, $>$ Fig.3, $>$ Fig.4, - Fig.5). Overall accuracy of SDSS-AI for identifying gastric anatomical landmarks was $94.2 \%$ (95\% Cl 92.9\%-95.2\%), with accuracies of $97.8 \% \quad(95 \% \mathrm{Cl} \quad 95.7 \%-$ $98.9 \%), 96.5 \%(95 \% \mathrm{Cl} 94.2 \%-98.0 \%)$, $73.8 \% \quad(95 \% \mathrm{Cl} \quad 69.2 \%-77.8 \%), \quad 96.0 \%$ (95\%Cl $93.6 \%-97.6 \%), \quad 98.0 \% \quad(95 \% \mathrm{Cl}$ $96.0 \%-99.1 \%), 96.0 \%(95 \% \mathrm{Cl} 93.6 \%-$ $97.6 \%), 96.8 \%(95 \% \mathrm{Cl} 94.5 \%-98.2 \%)$, and $98.8 \%$ (95\%Cl $97.0 \%-99.6 \%)$ for identifying cardia, fundus, body, greater curvature, lesser curvature, angulus, antrum and pylorus, respectively. Image processing time of the system was $94 \mathrm{~ms}$ per image ( $\triangleright$ Video 1 ).

In summary, SDSS-AI is a promising tool for real-time diagnosis and localization of gastric lesions in MCE examination, and aids physicians in improving lesion detection and avoiding blind spots. Further improvement of the deep learning system is needed, and studies with large sample sizes are warranted to evaluate the accuracy and efficacy of the system.

Endoscopy_UCTN_Code_CCL_1AG_2AF

Funding

Shanghai Municipal Hospital Emerging Frontier Technology Joint Project SHDC12019105

National Natural Science Foundation of China

81900600

“Ten Thousand Plan"-National High Level Talents Special Support Plan na 


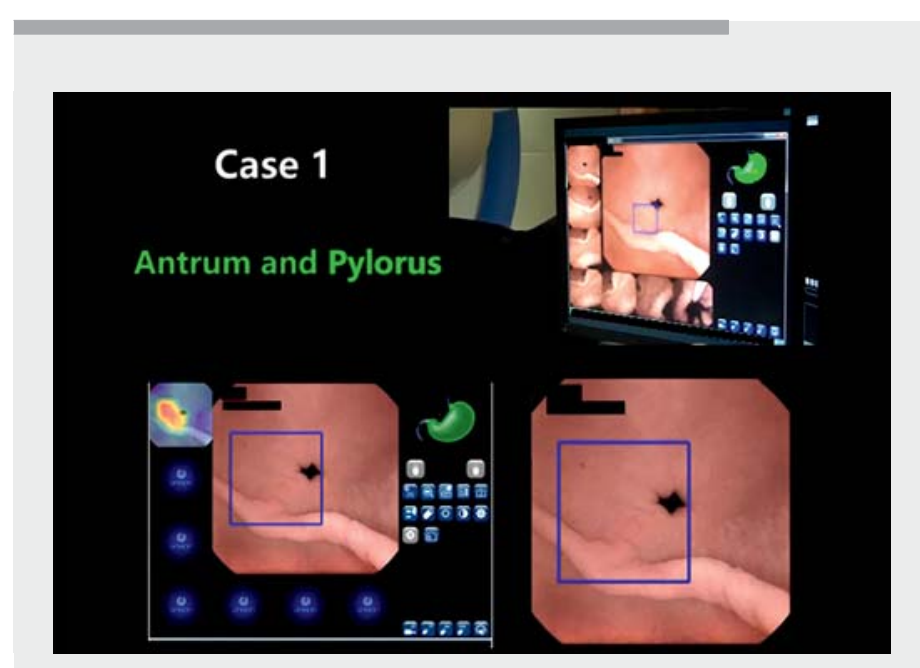

Video 1 Performance of the artificial intelligence-based real-time diagnostic system in magnetically controlled capsule endoscopy for identifying gastric lesions and anatomical landmarks.

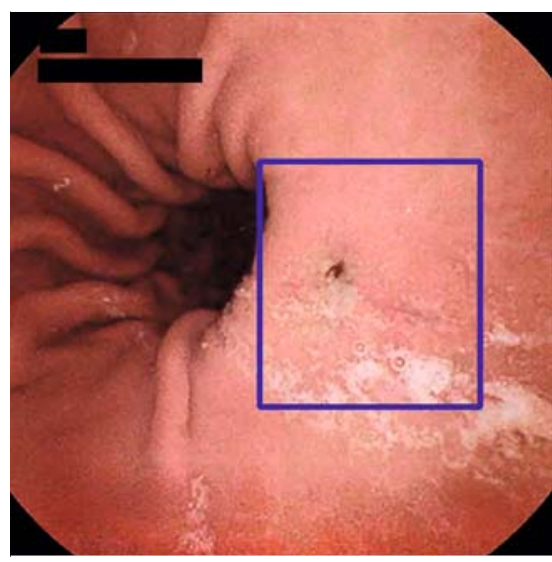

- Fig. 4 Real-time identification of erosion/bleeding/ulcer (blue frame) in the gastric body.

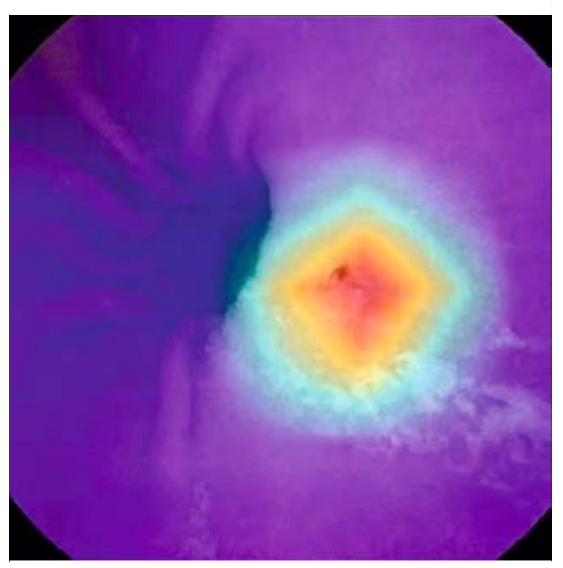

- Fig. 5 Lesion detection by heat map in the gastric body.

\section{Competing interests}

The authors declare that they have no conflict of interest.

The authors

Jun Pan ${ }^{1}$, Ji Xia ${ }^{1,2^{*}}$, Bin Jiang ${ }^{1,3}$, Hang Zhang ${ }^{4}$, Hao Zhang ${ }^{4}$, Zhao-Shen Li ${ }^{1}$, Zhuan Liao ${ }^{1} \odot$

1 National Clinical Research Center for Digestive Diseases, Department of Gastroenterology, Changhai Hospital, Shanghai, China

2 Department of Gastroenterology, No. 926 Hospital, Yunnan, China

3 Department of Gastroenterology, No. 422 Hospital, Guangdong, China

4 Ankon Technologies Co., Ltd., Wuhan, China

\section{Corresponding author}

\section{Zhuan Liao, MD}

National Clinical Research Center for Digestive Diseases, Department of Gastroenterology, Changhai Hospital, 168 Changhai Road, Shanghai 200433, China liaozhuan@smmu.edu.cn

\footnotetext{
* Drs. Pan and Xia contributed equally to this work.
}

[1] Xia J, Xia T, Pan J et al. Use of artificial intelligence for detection of gastric lesions by magnetically controlled capsule endoscopy. Gastrointest Endosc 2021; 93: 133-139

\section{Bibliography}

Endoscopy 2022; 54: E622-E623

DOI 10.1055/a-1724-6958

ISSN 0013-726X

published online 26.1.2022

(C) 2022. Thieme. All rights reserved.

Georg Thieme Verlag KG, Rüdigerstraße 14,

70469 Stuttgart, Germany

\section{ENDOSCOPY E-VIDEOS}

https://eref.thieme.de/e-videos

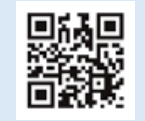

Endoscopy E-Videos is an open access online section, reporting on interesting cases and new techniques in gastroenterological endoscopy. All papers include a high quality video and all contributions are freely accessible online. Processing charges apply (currently EUR 375), discounts and wavers acc. to HINARI are available.

This section has its own submission website at

https://mc.manuscriptcentral.com/e-videos

\section{CORRECTION}

Real-time identification of gastric lesions and anatomical landmarks by artificial intelligence during magnetically controlled capsule endoscopy

Pan J, Xia J, Jiang B et al. Real-time identification of gastric lesions and anatomical landmarks by artificial intelligence during magnetically controlled capsule endoscopy. Endoscopy 2022, doi:10.1055/a-17246958

In the above-mentioned article, the authorship has been corrected. Drs. Pan and Xia are contributing equally. This was corrected in the online version on February 8, 2022. 